\title{
Correlation of $D L C 1$ gene methylation with oncogenic PIK3CA mutations in extramammary Paget's disease
}

\author{
Zhihua Kang ${ }^{1, *}$, Feng $\mathrm{Xu}^{2, *}$, Qiao-An Zhang ${ }^{2}$, Jinran $\mathrm{Lin}^{2}$, Zhiyuan $\mathrm{Wu}^{1}$, Xinju Zhang ${ }^{3}$, \\ Yan $\mathrm{Luo}^{2}$, Jinhua $\mathrm{Xu}^{2}$ and Ming Guan ${ }^{1,3}$ \\ ${ }^{1}$ Department of Laboratory Medicine, Huashan Hospital, Shanghai Medical School, Fudan University, \\ Shanghai, The People's Republic of China; ${ }^{2}$ Department of Dermatology, Huashan Hospital, Shanghai \\ Medical School, Fudan University, Shanghai, The People's Republic of China and ${ }^{3}$ Central Laboratory, \\ Huashan Hospital, Shanghai Medical School, Fudan University, Shanghai, The People's Republic of China
}

\begin{abstract}
Extramammary Paget's disease is a rare cutaneous malignant neoplasm. The genetic and epigenetic mechanisms underlying its pathology remain unknown. In this study, we investigated the expression levels, and mutation and methylation status of a common tumor suppressor gene, deleted in liver cancer 1 (DLC1), and an oncogene, PIK3CA, in tumor $(n=132)$ and normal tissues $(n=20)$ from unrelated patients. The presence of epigenetic and genetic lesions was then correlated to the patient pathology data to determine the potential role of these genes in extramammary Paget's disease etiology and progression. The DLC1 gene was found to be downregulated in $43(33 \%)$ tumors, as compared with immunohistochemistry results from normal tissues. Methylation-sensitive, high-resolution melting analysis indicated that the DLC1 promoter was hypermethylated in $51(39 \%)$ extramammary Paget's disease tumors. This hypermethylation was associated with significantly decreased DLC1 levels $(P=0.011)$, and had a strong positive correlation with advanced age $(P=0.002)$. PIK3CA mutations were detected by direct sequencing in $32(24 \%)$ tumors, the majority of which were invasive. Furthermore, PIK3CA mutations significantly correlated with DLC1 hypermethylation. Thus, aberrant DLC1 methylation and PIK3CA mutations may have important roles in extramammary Paget's disease pathogenesis, and may represent potential molecular targets for therapy.

Modern Pathology (2012) 25, 1160-1168; doi:10.1038/modpathol.2012.65; published online 20 April 2012
\end{abstract}

Keywords: DLC1, DNA methylation, extramammary Paget's disease; PIK3CA

Extramammary Paget's disease is a relatively rare cutaneous malignant neoplasm, which was first described by Crocker in $1889 .{ }^{1}$ Since then, many additional cases have been reported, providing general insights into the clinical and pathological characteristics of this rare condition. Extramammary Paget's disease most frequently involves skin with a high density of apocrine sweat glands, such as the

Correspondence: Professor M Guan, PhD, Department of Laboratory Medicine, Huashan Hospital, Shanghai Medical School, Fudan University, 12 Central Urumqi Road, Shanghai 200040, P.R. China or Professor J Xu, MD, Department of Dermatology, Huashan Hospital, Shanghai Medical School, Fudan University, 12 Central Urumqi Road, Shanghai 200040, P.R. China.

Email: guanming88@yahoo.com or xjhhs@medmail.com.cn

*These two authors contributed equally to this study.

Received 26 December 2011; revised 27 February 2012; accepted 27 February 2012; published online 20 April 2012 genitoperineal region and axilla, but cases have also been reported for other skin regions and mucosa. ${ }^{2}$ Clinical presentation often includes pruritus and an eczema-like lesion on the surface of the involved skin region. ${ }^{3}$ Epidemiological studies have reported that the majority of extramammary Paget's disease patients are older adults, ranging in age from 50 to 80 years old and having an average age of 65 years. ${ }^{4}$ Caucasian female patients are the most frequently reported population; however, in Asian populations, there is a male predominance for this disease. ${ }^{4,5}$ Although some familial extramammary Paget's disease cases have been reported ${ }^{6}$ the epigenetic and genetic abnormalities that contribute to the pathogenesis of this rare disease remain poorly understood.

The deleted in liver cancer 1 ( $D L C 1)$ gene, located on chromosome $8 \mathrm{p} 21-8 \mathrm{p} 22$, encodes a regulator of 
the Rho family of small GTPases. ${ }^{7}$ The principal function of DLC1 is to catalyze the conversion of active guanosine triphosphate-bound RhoA to the inactive guanosine biphosphate-bound form. As this enzymatic mechanism results in a negative regulatory effect on cell growth and tumorigenesis, DLC1 is considered a tumor suppressor gene. ${ }^{8,9}$ Loss of DLC1 expression, due to chromosomal deletion or promoter hypermethylation, has been detected in $50 \%$ of human hepatocellular carcinomas, and in many other human malignancies, including breast, prostate, colon, and lung. ${ }^{10-13}$ Accordingly, therapeutic upregulation of DLC1 expression in cancer cells has been proposed as a novel method to induce apoptosis, and inhibit cell proliferation and colony formation, ${ }^{14,15}$ and to restrain cell migration and suppress metastasis. ${ }^{16-18}$

A key factor in the phosphatidylinositol-3-kinase (PI3K)/AKT signaling pathway is PIK3CA. The PIK3CA gene mutations have been implicated in perturbed events of cell metabolism, growth, and survival. ${ }^{19-22}$ Several somatic mutations in the PIK3CA locus have recently been identified and found to occur at significant frequencies in various types of human cancer. ${ }^{23-26}$ More than $80 \%$ of these mutations are clustered in exon 9, which encodes the protein's helical domain, and in exon 20, which encodes the kinase domain. ${ }^{27}$ However, correlation analysis of the PIK3CA genetic alterations to determine their clinicopathological significance for extramammary Paget's disease has not yet been reported in the literature.

A recent study identified a statistically significant association between increased PIK3CA levels in tumors and promoter methylation of some tumor suppressor genes. ${ }^{28}$ In addition, previous evidence revealed that the activated PI3K/AKT pathway could negatively regulate the expression of DLC1 protein in tumors derived from hepatocyte-specific PIK3CA transgenic mice. ${ }^{29}$ Thus, a functional interaction likely exists between DLC1 silencing and PI3K/AKT signaling activation in tumor cells.

The aim of our study, therefore, was to investigate the methylation status of DLC1 and the presence of PIK3CA mutations in extramammary Paget's disease patients. The results of our study not only provide novel insights into the pathogenic roles of epigenetic and genetic events in extramammary Paget's disease, but also correlate such events with the clinicopathological features of the disease.

\section{Materials and methods}

\section{Clinical Samples}

Tumor tissues were biopsied from 132 unrelated patients diagnosed with extramammary Paget's disease, and treated in the Dermatology Department of Huashan Hospital (Shanghai, China) between 2005 and 2010. The patient population consisted of 110 males and 22 females, with an average age of
69.1 years (range: 47-92). The study protocol was approved by the Ethics Committee of Huashan Hospital, and all study participants provided written informed consent before study inclusion. The clinical and demographic data of all patients were reviewed by two dermatologists. To confirm the pre-surgery extramammary Paget's disease diagnosis, the histological sections from all cases were evaluated by hematoxylin and eosin staining to detect Paget cells, which are large, irregularly shaped cells with clear cytoplasm that are the key feature of extramammary Paget's disease ${ }^{30}$ In addition, histological examination of sequential tumor sections was used to confirm that each specimen contained more than 50\% tumor cells. The histological features of invasion were used to stratify the extramammary Paget's disease cases as noninvasive $(n=94)$ or invasive $(n=38)$. Normal skin specimens $(n=20)$ were collected from the penises of 20 of the extramammary Paget's disease patients (age-, and ethnicity-matched with the patients described above) by peritomy, and were used as normal noncancerous tissue controls.

\section{Methylation-Sensitive, High-Resolution Melting Analysis}

Genomic DNA was extracted from each formalinfixed, paraffin-embedded dermatic biopsy section by using the QIAamp DNA FFPE Tissue Kit (Qiagen, Hilden, Germany). The purified DNA was chemically modified by using the EpiTect Bisulfite Kit (Qiagen) according to the manufacturer's instructions, to detect methylation sites. Briefly, DNA exposure to sodium bisulfite converted all unmethylated cytosines to uracils, whereas leaving methylated cytosines unaltered.

A methylated reference sample, the CpGenome Universal methylated human male genomic DNA (Chemicon, Billerica, MA, USA), and an unmethylated reference sample, genomic DNA isolated from the peripheral blood mononuclear cells of a healthy male individual, were subjected to the bisulfite modification procedure and used as control standards. To create a standard dilution range of methylated and unmethylated alleles, the above two controls were mixed in $0,1,10,30,50,80$, and $100 \%$ ratios of methylated to unmethylated references. Each of the methylation-sensitive, highresolution melting analysis experimental runs included these seven methylated to unmethylated standard dilutions. The PCR amplification and highresolution melting analysis were carried out sequentially. The PCR amplification reaction mixture $(20 \mu \mathrm{l})$ was composed of $50 \mathrm{ng}$ bisulfate-treated template, $10 \mu \mathrm{l}$ Premix Taq Hot Start polymerase (TaKaRa, Dalian, China); $0.25 \mathrm{mM}$ primers (forward: 5'-TCGTTACGGTTTTAGAAAGAAA- $3^{\prime}$ and reverse: $5^{\prime}$-TTCGCTCCCAACCAAAACATAA- ${ }^{\prime}$ ), and $5 \mathrm{mM}$ SYTO-9 intercalating dye (Invitrogen, Carlsbad, CA, 
USA). The thermal-cycling reaction was performed on a 9700 GeneAmp PCR system (Applied Biosystems, Carlsbad, CA, USA) using the following conditions: 40 cycles of $30 \mathrm{~s}$ at $95{ }^{\circ} \mathrm{C}, 30 \mathrm{~s}$ at $55^{\circ} \mathrm{C}$, and $30 \mathrm{~s}$ at $72{ }^{\circ} \mathrm{C}$, and a final extension at $72{ }^{\circ} \mathrm{C}$ for 5 min. Immediately after the PCR amplification, high-resolution melting analysis was performed on a Roter-Gene Q real-time PCR cycler (Qiagen) using a $+0.1^{\circ} \mathrm{C} / \mathrm{s}$ temperature-ramping gradient from 72 to $90{ }^{\circ} \mathrm{C}$. Fluorescence acquisition was set as recommended by the manufacturer. The DLC1 methylation level was quantitated by analyzing the PCR-product melting curves with the accompanying Rotor-Gene Q software.

\section{Bisulfite DNA Sequencing}

PCR primers were designed as previously described by Guan et al, ${ }^{11}$ to amplify a $292 \mathrm{bp}$ region of the DLC1 promoter that encompasses 35 CpGs (Figure 1a). The PCR product was then subcloned into the pMD19-T expression vector by using a TA Cloning Kit (TaKaRa). Recombinant plasmids were sent to MAP Biotech (Shanghai, China) to complete the bisulfite DNA-sequencing procedure.

\section{Immunohistochemistry}

Immunohistochemical (IHC) studies were performed on 5 - $\mu$ m-thick sections of formalin-fixed, paraffin-embedded extramammary Paget's disease and normal tissue samples using standard techniques. The primary antibodies used targeted cytokeratin 7 (CK7, 1:100 dilution; Dakocytomation, Carpinteria, CA, USA), carcinoembryonic antigen (CEA, 1:100; Dakocytomation), and DLC1 (1:200; Santa Cruz Biotechnology, Santa Cruz, CA, USA). Biotinylated secondary antibodies of rabbit anti-mouse IgG (1:50) and HRP enzyme conjugate (all from MultiScience Biotech, Shanghai, China) were used to amplify and detect immunoreactivity. The 3,3'-diaminobenzidine chromogen substrate was used to visualize the results. All IHC analyses were carried out by a technician who was blinded to the clinical information. The CK7 and CEA expression (in cytoplasm and on the cell membrane) was defined as 'positive' if $>10 \%$ of the cells showed immunostaining. Cytoplasmic expression of DLC1 was defined as 'reduced' if $<25 \%$ of the cells were immunostained; otherwise, the sample was defined as having 'normal' DLC1 expression.

\section{PIK3CA Mutation Analysis}

Purified genomic DNA from all samples were subjected to PCR to amplify the PIK3CA exon 9 and exon 20 regions, according to the method previously described by Moroni et al. ${ }^{31}$ The PCR products were sent to MAP Biotech for direct DNA sequencing to detect the mutation status in each exon region for each sample.

\section{Statistical Analysis}

The $\chi^{2}$-test and Fisher's exact test were used to evaluate the association of DLC1 methylation status and PIK3CA mutations with the patients' clinical data. All statistical procedures were carried out by using the STATA 10.0 software package (StataCorp, College Station, TX, USA). A two-sided $P$-value of $<0.05$ was set as the threshold for statistical significance.

\section{Results}

\section{Methylation Levels of DLC1 CpG Islands in Extramammary Paget's Disease Tumors}

To evaluate the DLC1 methylation levels in extramammary Paget's disease tumors, we examined promoter hypermethylation by using the methylation-sensitive, high-resolution melting procedure. Methylation-sensitive, high-resolution melting analysis was implemented by initially constructing a series of normalized melting profiles for samples with different ratios of methylated to unmethylated template. These profiles were then used to generate a standard curve by which the methylation status of the experimental samples could be quantified (Figure 2). Using this method, 51 (39\%) of the total 132 extramammary Paget's disease tumor samples that were methylated on the $D L C 1$ promoter were identified. Moreover, the samples with methylation had $>1 \%$ of the promoter sequence methylated. Methylation-sensitive, high-resolution melting analysis of the 20 normal skin DNA samples detected no methylation in the DLC1 promoter. Therefore, the cancer samples were sub-classified into two groups: methylation-negative $(<1 \%$ methylation $)$ and methylation-positive (1-100\% methylation). Correlation analysis of the two DLC1 methylation level subgroups indicated that a higher methylation level was significantly associated with advanced patient age $(P=0.002$; Table 1$)$. In contrast, methylation level was not significantly associated with sex or extramammary Paget's disease invasion $(P=0.729$ and $P=0.360$, respectively; Table 1 ).

\section{Bisulfite DNA Sequencing}

Bisulfite DNA sequencing was employed to determine the comprehensive extramammary Paget'sdisease-related methylation pattern of the $5^{\prime}-\mathrm{CpG}$ islands in the $D L C 1$ promoter. A 292-bp fragment in the $D L C 1$ promoter, covering $35 \mathrm{CpG}$ dinucleotides, was amplified from a set of 6 extramammary Paget's disease samples that were identified by methylation-sensitive, high-resolution melting analysis as methylated and from a normal skin tissue sample (N1). Bisulfite DNA sequencing showed that the 
a

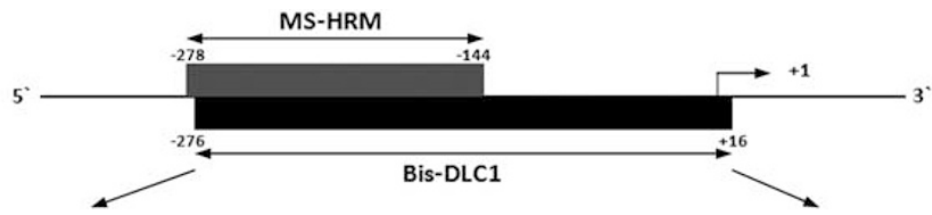

GCTCCCAGCCAGGACATGGCCGCACCTCTCCGCATCAGGAGCGCCGGCTCACGGACTTCTCGCCC AACTCCCTGAGCGCTCCCTCGTTTCGATCTTTAGAAAACCCCGCTTTCTTTCTGGGGCCGTGACG AGGGGCAGGGAGCGGCGAGCAAGGATTCGTTGAGGACCGCGAGGGCGCGCGTCTCGGGTGCCGCC GTGGGTCCCGACGCG 13 AAAGCCGAGCCGCCTCCGCCTGCCTCGACTTCCCCACAGCGCTTCCGCCG CCGCCTGCCGTGCTTGATGTGCAGAAAGAAGC 29 34

b

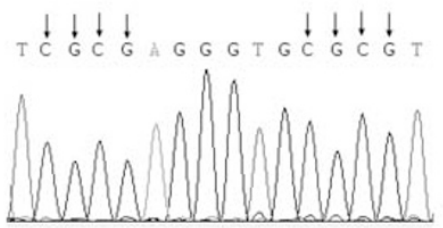

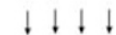

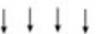

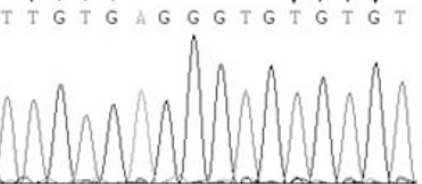

c

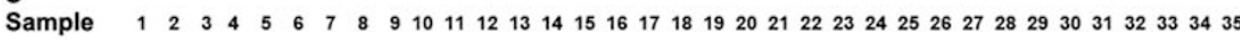
00000000000000000000000000000000000 00000000000000000000000000000000000 O O O OOOOOOOOOOOOOOOOOOOOOOOOOOOOOOOO

S3

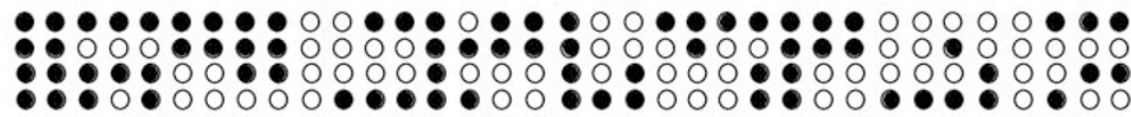

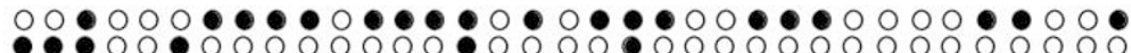

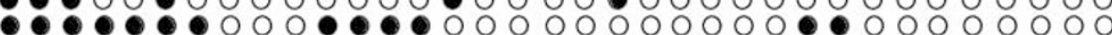

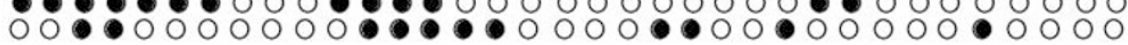

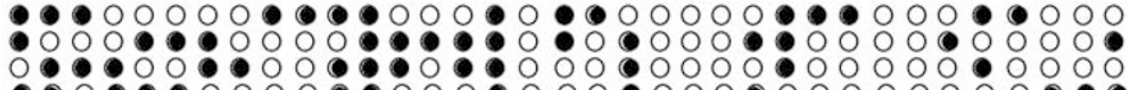

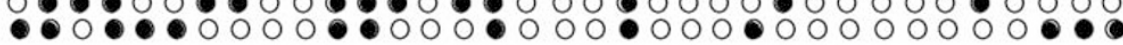

$\bullet \bullet \bullet \bullet \bullet \circ \bullet \circ \circ \circ \circ \bullet \bullet \circ$ 1000

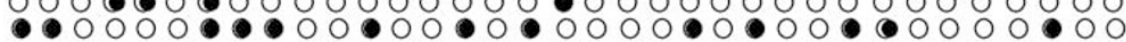

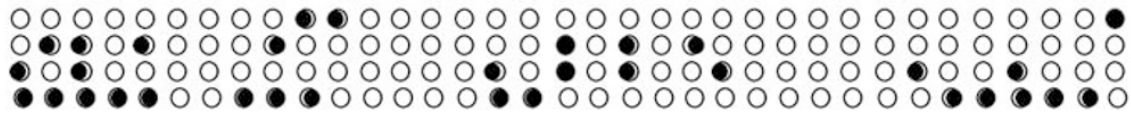

Figure 1 Methylation status of the CpG island region in the deleted in liver cancer 1 (DLC1) promoter of extramammary Paget's disease patients. (a) Schematic depiction of the DLC1 promoter-associated CpG island, which spans the region from -278 to +16 , with respect to the ATG start site $(+1)$. Regions analyzed by MS-HRM and bisulfite genomic sequencing (Bis-DLC1) are shown. The Bis-DLC1 region encompassed 292 bp and contained 35 CpG dinucleotides. (b) Examples of a highly methylated DLC1 CpG island (sample S3, left) and an unmethylated $\mathrm{CpG}$ island (sample N1, right), as determined by bisulfite sequencing analysis. Arrows indicate the positions of CpG dinucleotides. (c) Methylation patterns of the Bis-DLC1 region of the DLC1 CpG island in a normal tissue sample, and six extramammary Paget's disease tumors and hyperplasia samples that were identified by MS-HRM as methylated. Methylated and unmethylated CpG sites are shown as solid circles and open circles, respectively.

CpG islands in normal skin samples were unmethylated, and that there was frequent methylation in the six extramammary Paget's disease samples (Figure 1c). Although each of the six tumor samples shared some common methylation sites, the overall methylation patterns were distinct.

\section{IHC Expression}

The CK7 and CEA expression are key pathological features of Paget cells, and IHC detection of these two proteins is generally considered an accurate diagnostic method for extramammary Paget's 


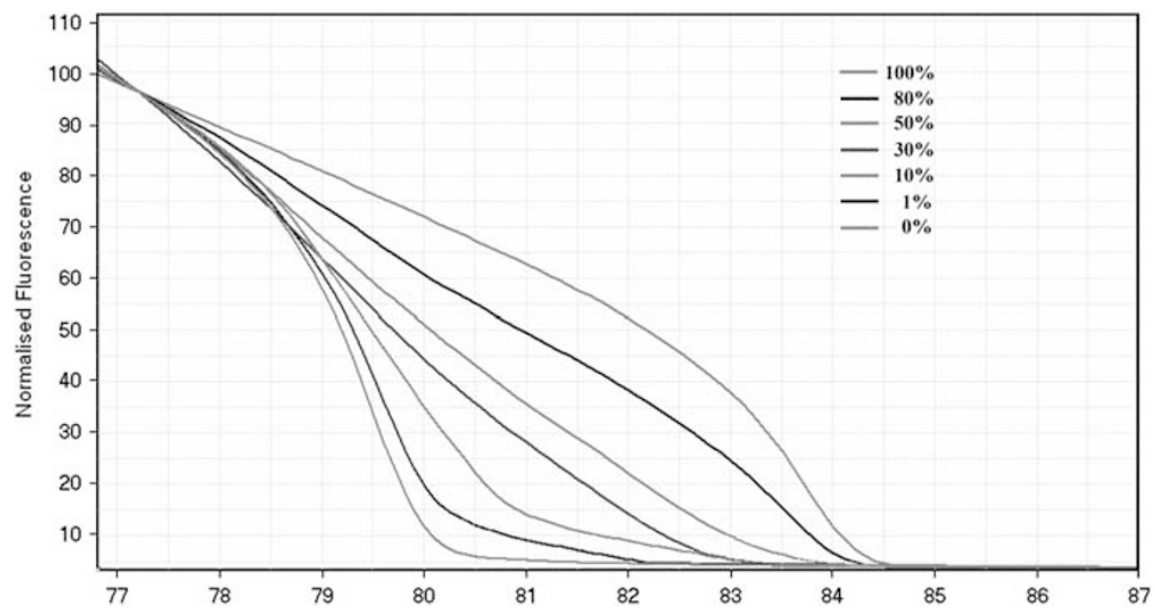

Figure 2 The normalized methylation-sensitive, high-resolution melting analysis standard curve used to evaluate methylation levels of extramammary Paget's disease cases.

Table 1 DLC1 methylation and protein levels in extramammary Paget's disease

\begin{tabular}{|c|c|c|c|c|c|c|c|}
\hline & \multirow[t]{2}{*}{ Total } & \multicolumn{2}{|c|}{$\begin{array}{l}\text { Methylation } \\
\text { level }^{\mathrm{a}}\end{array}$} & \multirow[t]{2}{*}{$\mathrm{P}$-value ${ }^{\mathrm{b}}$} & \multicolumn{2}{|c|}{$\begin{array}{l}\text { Protein } \\
\text { level }^{\mathrm{c}}\end{array}$} & \multirow[t]{2}{*}{$\mathrm{P}$-value } \\
\hline & & $0-1 \%$ & $1-100 \%$ & & Normal & Reduced & \\
\hline Case, $n$ & 132 & 81 & 51 & - & 89 & 43 & - \\
\hline \multicolumn{8}{|l|}{ Age, years } \\
\hline$>70$ & 60 & 26 & 31 & 0.002 & 34 & 26 & 0.016 \\
\hline$\leq 70$ & 72 & 52 & 20 & - & 55 & 17 & - \\
\hline \multicolumn{8}{|l|}{ Sex } \\
\hline Male & 110 & 67 & 43 & 0.729 & 78 & 38 & 0.352 \\
\hline Female & 22 & 13 & 7 & - & 17 & 5 & - \\
\hline \multicolumn{8}{|c|}{ Invasion status } \\
\hline Invasive & 38 & 21 & 17 & 0.360 & 23 & 15 & 0.282 \\
\hline In situ & 94 & 60 & 34 & - & 66 & 28 & - \\
\hline
\end{tabular}

Abbreviations: DLC1, deleted in liver cancer 1; IHC, immunohistochemistry.

${ }^{\mathrm{a}}$ Detected by methylation-sensitive, high-resolution melting analysis.

${ }^{\mathrm{b}}$ Determined by $\chi^{2}$-test.

${ }^{\mathrm{c}}$ Detected by IHC.

disease. In our study, all extramammary Paget's disease samples showed positive CK7 and CEA staining by IHC (Figure 3). In addition, the IHC findings were used to evaluate the potential correlation between DLC1 protein expression and the methylation status of the DLC1 promoter. The IHC staining indicated that DLC1 was reduced in 43 $(33 \%)$ of the 132 tumor samples. Moreover, a significant correlation was found between DNA methylation of $\mathrm{CpG}$ islands in the DLC1 promoter and reduced DLC1 protein expression in the extramammary Paget's disease tumor samples $(P=0.011$; Table 2).

Strong immunostaining for DLC1 was observed in all normal skin tissue samples. These samples were considered to represent the baseline level of DLC1 expression, and all of these samples exhibited an absolute lack of methylation in the DLC1 promoter region. In contrast, the tumor tissues were heterogeneous, having methylated and unmethylated DLC1 alleles (Figure 1a).

\section{PIK3CA Mutations in Extramammary Paget's Disease}

To determine the prevalence of PIK3CA mutations in extramammary Paget's disease patients, we analyzed the PIK3CA exon 9 and exon 20 coding sequences, both of which are known as mutation hotspots and include the frequently mutated codons 542, 545 , and 1047. Of the 35 mutations that were detected in PIK3CA of the 132 patients in our study, 25 cases were detected in exon 9, which encodes the helical domain of PI3K ( 5 cases with c.1624G $>$ A, 1 c. $1633 \mathrm{G}>\mathrm{A}$, and 20 c. $1634 \mathrm{~A}>\mathrm{C}$ ), and 10 cases were detected in exon 20, which encodes the catalytic domain of PI3K (2 c.3296C $>$ T and 8 c.3297A > G; Table 3). Although mutations were found in only 32 $(24 \%)$ of the extramammary Paget's disease cases, the correlation between PIK3CA mutations and DLC1 methylation was significant $(P=0.001)$. In addition, PIK3CA mutations were found to be significantly associated with invasive extramammary Paget's disease $(P=0.006)$.

\section{Discussion}

The rarity of extramammary Paget's disease has precluded its comprehensive characterization. As the majority of studies to date have focused on IHC detection of one or several proteins, ${ }^{32,33}$ our knowledge of the genetic and epigenetic features of extramammary Paget's disease remain largely unknown. In this study, we collected extramammary Paget's disease tumor samples from 132 unrelated 

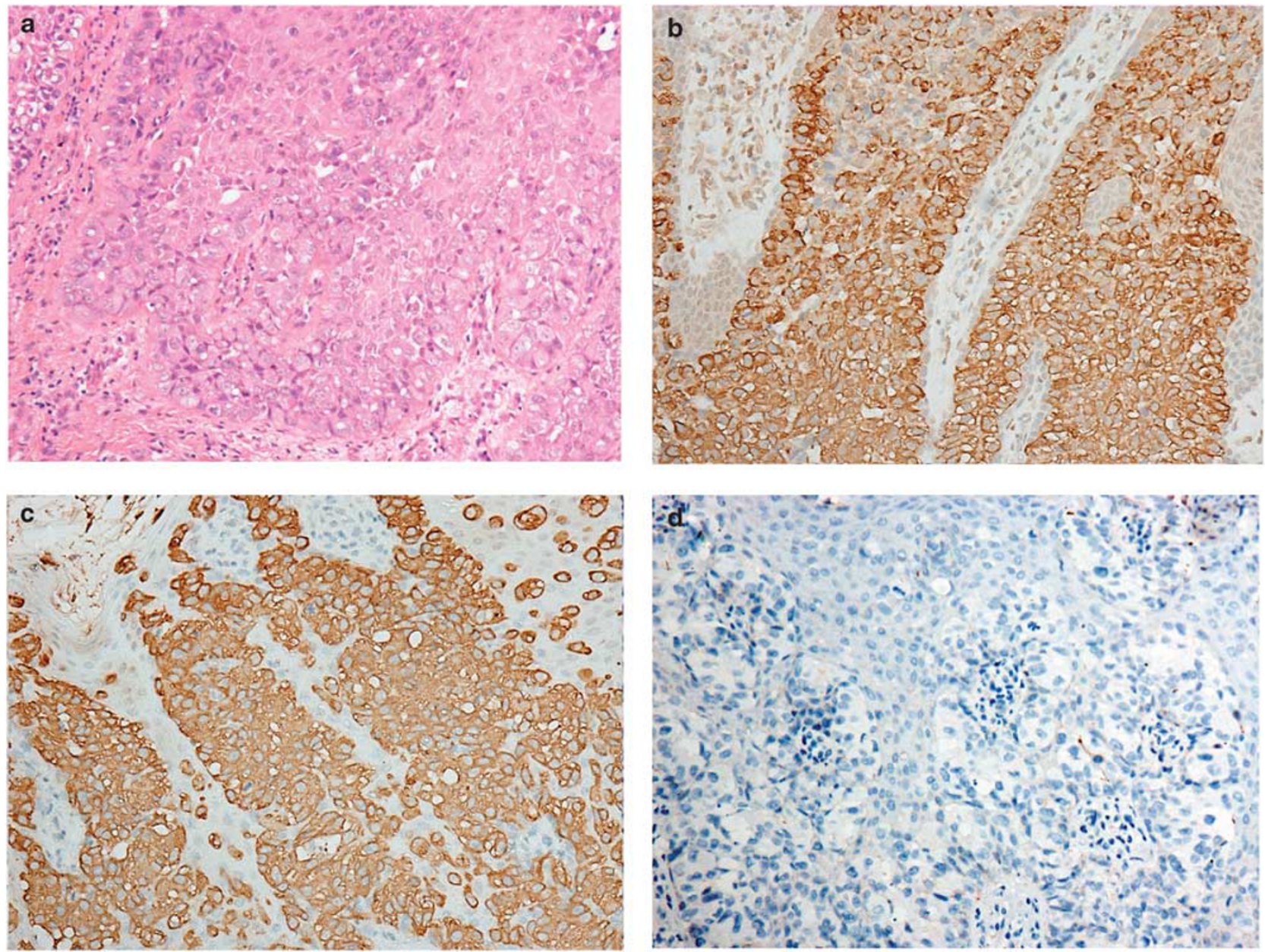

Figure 3 Immunohistochemical analysis of the deleted in liver cancer 1 (DLC1) protein expression in extramammary Paget's disease. (a) Extramammary Paget's disease skin pathological section stained with hematoxylin and eosin staining $(\times 200)$. (b) DLC1 protein expression in a normal skin tissue was localized to the cytoplasm $(\times 200)$. (c) DLC1 protein expression in an extramammary Paget's disease tissue with a $0 \%$ methylation level was localized to the cytoplasm $(\times 200)$. (d) DLC1 protein expression was completely undetectable in an extramammary Paget's disease tissue with $50-80 \%$ methylation $(\times 200)$.

Table 2 DLC1 methylation and protein expression levels in extramammary Paget's disease patients with PIK3CA mutations

\begin{tabular}{|c|c|c|c|c|c|c|}
\hline \multirow[t]{2}{*}{$\begin{array}{l}\text { Methylation } \\
\text { level }\end{array}$} & \multicolumn{2}{|c|}{$\begin{array}{l}\text { DLC1 } \\
\text { protein }\end{array}$} & \multirow[t]{2}{*}{ P-value } & \multicolumn{2}{|c|}{$\begin{array}{l}\text { PIK3CA } \\
\text { mutations }\end{array}$} & \multirow[t]{2}{*}{$\mathrm{P}$-value } \\
\hline & Reduced & Normal & & Positive & Negative & \\
\hline$>1 \%$ & 23 & 28 & 0.011 & 20 & 31 & 0.001 \\
\hline$<1 \%$ & 20 & 63 & & 12 & 69 & \\
\hline
\end{tabular}

Abbreviation: DLC1, deleted in liver cancer 1.

${ }^{\mathrm{a}} \chi^{2}$-test.

patients over a 5-year period. Our China-based study population accurately reflected the previously reported male bias for extramammary Paget's disease in Asians; specifically, our patients represented a male to female ratio of 1:5. To the best of our knowledge, the set of extramammary Paget's disease patients collected for evaluation from our institute represents the largest set ever reported on in a single study. Twenty normal skin tissues were collected from the same patients with extramammary Paget's disease; it is important to note that this small number of controls, as compared with the experimental sample number, represents a limitation of the present study.

In this study, we found a high prevalence of downregulation and hypermethylation of the DLC1 gene in cases of this rare disease (33 and 39\%, respectively). In addition, activating PIK3CA mutations were detected in $24 \%$ of the extramammary Paget's disease cases. Taken together, these findings provide solid evidence that epigenetic and genetic alterations are common in extramammary Paget's disease and may be involved in the disease pathogenesis.

Rho GTPases are proteins with pleiotropic cellular functions, including the regulation of actin 
Table 3 PIK3CA mutations detected in extramammary Paget's disease cases

\begin{tabular}{|c|c|c|c|c|}
\hline \multirow[t]{2}{*}{ Case } & \multirow[t]{2}{*}{ Age/sex } & \multirow[t]{2}{*}{ Tumor } & \multicolumn{2}{|c|}{ PIK3CA mutations } \\
\hline & & & Nucleotide mutation & Amino acid substitution \\
\hline 1 & $74 / \mathrm{F}$ & In situ & c. $3297 \mathrm{~A}>\mathrm{G}$ & p.His1047Arg \\
\hline 2 & $72 / \mathrm{M}$ & In situ & c. $1634 \mathrm{~A}>\mathrm{C}$ & p.Glu545Ala \\
\hline 3 & $84 / F$ & In situ & c. $1624 \mathrm{G}>\mathrm{A}$ & p.Glu542Lys \\
\hline 4 & $79 / \mathrm{M}$ & In situ & c. $1634 \mathrm{~A}>\mathrm{C}$ & p.Glu545Ala \\
\hline 5 & $63 / \mathrm{M}$ & In situ & c. $1624 \mathrm{G}>\mathrm{A}$, с. $1634 \mathrm{~A}>\mathrm{C}$ & p.Glu542Lys, p.Glu545Ala \\
\hline 6 & $59 / F$ & In situ & c. $1634 \mathrm{~A}>\mathrm{C}$ & p.Glu545Ala \\
\hline 7 & $78 / \mathrm{M}$ & In situ & c. $1634 \mathrm{~A}>\mathrm{C}$ & p.Glu545Ala \\
\hline 8 & $54 / \mathrm{M}$ & In situ & c. $3297 A>G$ & p.His1047Arg \\
\hline 9 & $70 / \mathrm{M}$ & In situ & c. $1634 \mathrm{~A}>\mathrm{C}$ & p.Glu545Ala \\
\hline 10 & $63 / \mathrm{M}$ & In situ & c. $1634 \mathrm{~A}>\mathrm{C}$, с. $3297 \mathrm{~A}>\mathrm{G}$ & p.Glu545Ala, p.His1047Arg \\
\hline 11 & $76 / \mathrm{M}$ & In situ & c. $1634 \mathrm{~A}>\mathrm{C}$ & p.Glu545Ala \\
\hline 12 & $79 / \mathrm{M}$ & In situ & c. $1634 \mathrm{~A}>\mathrm{C}$ & p.Glu545Ala \\
\hline 13 & $63 / \mathrm{M}$ & In situ & c. $1634 \mathrm{~A}>\mathrm{C}$ & p.Glu545Ala \\
\hline 14 & $81 / \mathrm{M}$ & In situ & c. $1634 \mathrm{~A}>\mathrm{C}$ & p.Glu545Ala \\
\hline 15 & $73 / \mathrm{M}$ & In situ & c. $1634 \mathrm{~A}>\mathrm{C}$ & p.Glu545Ala \\
\hline 16 & $79 / \mathrm{M}$ & In situ & c. $1634 \mathrm{~A}>\mathrm{C}$ & p.Glu545Ala \\
\hline 17 & $72 / \mathrm{M}$ & In situ & c. $1624 \mathrm{G}>\mathrm{A}$ & p.Glu542Lys \\
\hline 18 & $54 / \mathrm{M}$ & Invasive & c. $1634 \mathrm{~A}>\mathrm{C}$ & p.Glu545Ala \\
\hline 19 & $59 / \mathrm{M}$ & Invasive & c. $1634 \mathrm{~A}>\mathrm{C}$ & p.Glu545Ala \\
\hline 20 & $75 / \mathrm{M}$ & Invasive & c. $1634 \mathrm{~A}>\mathrm{C}$, с. $3296 \mathrm{C}>\mathrm{T}$ & p.Glu545Ala, p.His1047Tyr \\
\hline 21 & $58 / \mathrm{M}$ & Invasive & c. $1633 \mathrm{G}>\mathrm{A}$ & p.Glu545Lys \\
\hline 22 & $70 / \mathrm{M}$ & Invasive & c. $3297 \mathrm{~A}>\mathrm{G}$ & p.His1047Arg \\
\hline 23 & $58 / \mathrm{M}$ & Invasive & c. $1634 \mathrm{~A}>\mathrm{C}$, с. $3297 \mathrm{~A}>\mathrm{G}$ & p.Glu545Ala, p. His1047Arg \\
\hline 24 & $89 / \mathrm{M}$ & Invasive & c. $3297 A>G$ & p.His1047Arg \\
\hline 25 & $75 / \mathrm{M}$ & Invasive & c. $3296 \mathrm{C}>\mathrm{T}$ & p.His1047Tyr \\
\hline 26 & $81 / \mathrm{M}$ & Invasive & c. $1624 \mathrm{G}>\mathrm{A}$ & p.Glu542Lys \\
\hline 27 & $70 / \mathrm{M}$ & Invasive & c. $3297 A>G$ & p.His1047Arg \\
\hline 28 & $62 / \mathrm{M}$ & Invasive & c. $1634 \mathrm{~A}>\mathrm{C}$ & p.Glu545Ala \\
\hline 29 & $80 / \mathrm{M}$ & Invasive & c. $1634 \mathrm{~A}>\mathrm{C}$ & p.Glu545Ala \\
\hline 30 & $62 / \mathrm{F}$ & Invasive & c. $1634 \mathrm{~A}>\mathrm{C}$ & p.Glu545Ala \\
\hline 31 & $66 / \mathrm{M}$ & Invasive & c. $3297 \mathrm{~A}>\mathrm{G}$ & p.His1047Arg \\
\hline 32 & $79 / \mathrm{M}$ & Invasive & c. $1624 \mathrm{G}>\mathrm{A}$ & p.Glu542Lys \\
\hline
\end{tabular}

Abbreviations: F, female; M, male.

cytoskeletal dynamics, proliferation, migration, and invasion. Thus, deregulation of Rho activity is involved in oncogenic transformation of cells. ${ }^{34}$ Accumulating evidence has recently indicated that DLC1 may act as a tumor suppressor by inhibiting Rho signaling. Moreover, loss of DLC1 expression has been clinically detected in many types of human tumors. Herein, we reported that reduced DLC1 expression significantly correlated with $D L C 1$ promoter methylation $(P=0.011)$. Surprisingly, our data also showed that hypermethylation of DLC1 occurred preferentially in older subjects ( $>70$ years of age). Given the known association between aging and extramammary Paget's disease, ${ }^{3,35}$ we speculate that DLC1 methylation has an active role in neoplastic transformation. It is possible that agedependent methylation events involving the DLC1 promoter may represent an important underlying mechanism that links aging and extramammary Paget's disease. Nonetheless, our findings demonstrated that epigenetic abnormality of the DLC1 promoter (hypermethylation) is a common and major mechanism for the silencing of this gene in extramammary Paget's disease, and may represent an important oncogenic inducer.
Previous studies have suggested that persistent activation of AKT is a frequent finding in extramammary Paget's disease. ${ }^{36,37}$ Presently, we detected PIK3CA mutations in a large series of extramammary Paget's disease tissue samples to investigate whether the mutation is involved in the pathogenesis of this disease. Our data indicated that $24 \%$ of patients had PIK3CA mutations, suggesting that the PI3K/AKT pathway is constitutively activated in these individuals and might contribute to the development of extramammary Paget's disease. This finding led us to focus our attention on the role of PIK3CA mutations in the etiology and progression of extramammary Paget's disease. By performing a correlative analysis of the clinical data of patients with the PIK3CA mutation status, we found that individuals harboring PIK3CA mutations are more likely to develop invasive extramammary Paget's disease. It has been reported that dermal invasion in extramammary Paget's disease may be the most significant risk factor for death. ${ }^{38}$ Unfortunately, we do not have follow-up data on each of our extramammary Paget's disease patients and are unable to determine the survival rates of those with invasive extramammary Paget's disease and PIK3CA mutations. 
Nonetheless, a key biological implication of our study is that targeted molecular therapies to inhibit the PI3K/ATK signaling pathway may provide a new strategy for treating invasive extramammary Paget's disease.

As noted earlier in this report, nearly $80 \%$ of cancer-related PIK3CA mutations occur at three hotspots in the gene: Glu542Lys and Glu545Lys located within the helical domain, and His1047Arg located within the kinase domain. ${ }^{27}$ These mutations are known to result in elevated catalytic activity, which leads to oncogenic transformation. ${ }^{39}$ However, in our study, the Glu545Ala (c. $1634 \mathrm{~A}>$ C) mutation in exon 9 of PIK3CA showed a high frequency in PIK3CA mutation-positive extramammary Paget's disease cases (20 of 32), as compared with only 5 cases with the Glu542Lys, 1 case with Glu545Lys, 2 cases with His1047Tyr, and 8 cases with His1047Arg. To date, very few reports of human cancers have identified the Glu545Ala mutation. ${ }^{40-42}$ Yet, in our study, its frequency was even higher than the three mutation hotspots, suggesting that the Glu545Ala mutation may contribute to some distinct biological characteristics of Paget cells, possibly even supporting the distinctive properties of extramammary Paget's disease. However, it remains unknown precisely how the Glu545Ala mutation influences the pathogenesis and progression of Paget cells.

Kudo et $a l^{29}$ reported that DLC1 was downregulated in tumors derived from hepatocyte-specific PIK3CA transgenic mice, indicating that DLC1 is negatively regulated by activation of the PI3K/AKT pathway. Consistent with those findings, our results revealed that a strong correlation exists between PIK3CA mutation and DLC1 methylation $(P=0.001)$, highlighting the interaction between genetic and epigenetic alterations in extramammary Paget's disease, although, the mechanism underlying this phenomenon requires further study. On the other hand, overactivation of certain oncogenic pathways is known to affect the activity of methytransferases and regulation of gene transcription, possibly affecting components of the MAPK pathway, such as RAS, RAF, MEK, and ERK. ${ }^{43,44}$ For example, activating gene mutations in PIK3CA have been shown to lead to aberrant methylation and expression of the PTEN gene. ${ }^{45}$ Collectively, the results from our study and previous work by others suggest that epigenetic alterations of DLC1 might occur as a consequence of overactivation of the PI3K/AKT pathway in extramammary Paget's disease.

In conclusion, our study demonstrated that DLC1 promoter methylation occurs at a higher frequency in tumor tissues than in normal tissues from extramammary Paget's disease patients, and that downregulation of $D L C 1$ protein level strongly correlates with methylation of the DLC1 promoter. Importantly, we have revealed a significant association of DLC1 hypermethylation with PIK3CA mutations in extramammary Paget's disease. Activation of the PI3K/AKT pathway may have an important role in development of extramammary Paget's disease, and may represent a novel therapeutic target for treating this disease.

\section{Acknowledgements}

This study was supported by foundation from the National Key Discipline, Shanghai Municipal Health Bureau Hundred Talents Program (XBR2011044), and a grant from the Shanghai Science and Technology Commission (Grant No. 11DZ0512000). We thank Dr Nicholas C. Popescu for critical reading of the article. We thank Medjaden Bioscience Limited for assisting in the preparation of this manuscript.

\section{Disclosure/conflict of interest}

The authors declare no conflicts of interests.

\section{References}

1 Minicozzi A, Borzellino G, Momo R, et al. Perianal Paget's disease: presentation of six cases and literature review. Int J Colorectal Dis 2010;25:1-7.

2 Siesling S, Elferink MA, van Dijck JA, et al. Epidemiology and treatment of extramammary Paget disease in the Netherlands. Eur J Surg Oncol 2007;33: 951-955.

3 Shaco-Levy R, Bean SM, Vollmer RT, et al. Paget disease of the vulva: a study of 56 cases. Eur J Obstet Gynecol Reprod Biol 2010;149:86-91.

4 Lam C, Funaro D. Extramammary Paget's disease: summary of current knowledge. Dermatol Clin 2010; 28:807-826.

5 Kyriazanos ID, Stamos NP, Miliadis L, et al. Extramammary Paget's disease of the perianal region: a review of the literature emphasizing the operative management technique. Surg Oncol 2011;20:e61-e71.

6 Demitsu T, Gonda K, Tanita M, et al. Extramammary Paget's disease in two siblings. Br J Dermatol 1999;141: 951-953.

7 Xue W, Krasnitz A, Lucito R, et al. DLC1 is a chromosome 8p tumor suppressor whose loss promotes hepatocellular carcinoma. Genes Dev 2008;22: 1439-1444.

8 Wong CC, Wong CM, Ko FC, et al. Deleted in liver cancer 1 (DLC1) negatively regulates Rho/ROCK/MLC pathway in hepatocellular carcinoma. PloS One 2008;3:e2779.

9 Ullmannova-Benson V, Guan M, Zhou X, et al. DLC1 tumor suppressor gene inhibits migration and invasion of multiple myeloma cells through RhoA GTPase pathway. Leukemia 2009;23:383-390.

10 Yuan BZ, Miller MJ, Keck CL, et al. Cloning, characterization, and chromosomal localization of a gene frequently deleted in human liver cancer (DLC-1) homologous to rat RhoGAP. Cancer Res 1998;58: 2196-2199. 
11 Guan M, Zhou X, Soulitzis N, et al. Aberrant methylation and deacetylation of deleted in liver cancer-1 gene in prostate cancer: potential clinical applications. Clin Cancer Res 2006;12:1412-1419.

12 Ullmannova V, Popescu NC. Inhibition of cell proliferation, induction of apoptosis, reactivation of DLC1, and modulation of other gene expression by dietary flavone in breast cancer cell lines. Cancer Detect Prev 2007;31:110-118.

13 Yuan BZ, Durkin ME, Popescu NC. Promoter hypermethylation of DLC-1, a candidate tumor suppressor gene, in several common human cancers. Cancer Genet Cytogenet 2003;140:113-117.

14 Wong CM, Yam JW, Ching YP, et al. Rho GTPaseactivating protein deleted in liver cancer suppresses cell proliferation and invasion in hepatocellular carcinoma. Cancer Res 2005;65:8861-8868.

15 Loyo M, Brait M, Kim MS, et al. A survey of methylated candidate tumor suppressor genes in nasopharyngeal carcinoma. Int J Cancer 2011;128:1393-1403.

16 Healy KD, Hodgson L, Kim TY, et al. DLC-1 suppresses non-small cell lung cancer growth and invasion by RhoGAP-dependent and independent mechanisms. Mol Carcinog 2008;47:326-337.

17 Goodison S, Yuan J, Sloan D, et al. The RhoGAP protein DLC-1 functions as a metastasis suppressor in breast cancer cells. Cancer Res 2005;65:6042-6053.

18 Zhang $\mathrm{T}$, Zheng J, Jiang $\mathrm{N}$, et al. Overexpression of DLC-1 induces cell apoptosis and proliferation inhibition in the renal cell carcinoma. Cancer Lett 2009;283:59-67.

19 Samuels Y, Ericson K. Oncogenic PI3K and its role in cancer. Curr Opin Oncol 2006;18:77-82.

20 Bunney TD, Katan M. Phosphoinositide signalling in cancer: beyond PI3K and PTEN. Nat Rev Cancer 2010;10:342-352.

21 Duronio V, Scheid MP, Ettinger S. Downstream signalling events regulated by phosphatidylinositol 3-kinase activity. Cell Signal 1998;10:233-239.

22 Jiang BH, Zheng JZ, Aoki M, et al. Phosphatidylinositol 3-kinase signaling mediates angiogenesis and expression of vascular endothelial growth factor in endothelial cells. Proc Natl Acad Sci USA 2000;97: 1749-1753.

23 Jhawer M, Goel S, Wilson AJ, et al. PIK3CA mutation/ PTEN expression status predicts response of colon cancer cells to the epidermal growth factor receptor inhibitor cetuximab. Cancer Res 2008;68:1953-1961.

24 Dias-Santagata D, Lam Q, Bergethon K, et al. A potential role for targeted therapy in a subset of metastasizing adnexal carcinomas. Mod Pathol 2011;24: 974-982.

25 Troxell ML, Levine J, Beadling C, et al. High prevalence of PIK3CA/AKT pathway mutations in papillary neoplasms of the breast. Mod Pathol 2010;23: $27-37$.

26 Catasus L, Gallardo A, Cuatrecasas M, et al. Concomitant PI3K-AKT and p53 alterations in endometrial carcinomas are associated with poor prognosis. Mod Pathol 2009;22:522-529.

27 Meyer DS, Brinkhaus H, Muller U, et al. Luminal expression of PIK3CA mutant H1047R in the mammary gland induces heterogeneous tumors. Cancer Res 2011;71:4344-4351
28 Zuo T, Liu TM, Lan X, et al. Epigenetic silencing mediated through activated PI3K/AKT signaling in breast cancer. Cancer Res 2011;71:1752-1762.

29 Kudo Y, Tanaka Y, Tateishi K, et al. Altered composition of fatty acids exacerbates hepatotumorigenesis during activation of the phosphatidylinositol 3-kinase pathway. J Hepatol 2011;55:1400-1408.

30 Kohler S, Rouse RV, Smoller BR. The differential diagnosis of pagetoid cells in the epidermis. Mod Pathol 1998;11:79-92.

31 Moroni M, Veronese S, Benvenuti S, et al. Gene copy number for epidermal growth factor receptor (EGFR) and clinical response to antiEGFR treatment in colorectal cancer: a cohort study. Lancet Oncol 2005;6: 279-286.

32 Liegl B, Horn LC, Moinfar F. Androgen receptors are frequently expressed in mammary and extramammary Paget's disease. Mod Pathol 2005;18:1283-1288.

33 Ellis PE, Cano SD, Fear M, et al. Reduced E-cadherin expression correlates with disease progression in Paget's disease of the vulva but not Paget's disease of the breast. Mod Pathol 2008;21:1192-1199.

34 Holeiter G, Heering J, Erlmann P, et al. Deleted in liver cancer 1 controls cell migration through a Dia1dependent signaling pathway. Cancer Res 2008;68: 8743-8751.

35 Wagner G, Sachse MM. Extramammary Paget disease clinical appearance, pathogenesis, management. J Dtsch Dermatol Ges 2011;9:448-454.

36 Chen SY, Moroi Y, Urabe K, et al. Concordant overexpression of p-FAK and p-ERK1/2 in extramammary Paget's disease. Arch Dermatol Res 2008;300:195-201.

$37 \mathrm{Liu} \mathrm{H}$, Moroi Y, Yasumoto S, et al. Expression of insulin-like growth factor-1 receptor, p-AKT and p-ERK1/2 protein in extramammary Paget's disease. Br J Dermatol 2006;155:586-591.

38 Hatta N, Yamada M, Hirano T, et al. Extramammary Paget's disease: treatment, prognostic factors and outcome in 76 patients. Br J Dermatol 2008;158:313-318.

39 Kang S, Bader AG, Vogt PK. Phosphatidylinositol 3-kinase mutations identified in human cancer are oncogenic. Proc Natl Acad Sci USA 2005;102:802-807.

40 Teng HW, Wang HW, Chen WM, et al. Prevalence and prognostic influence of genomic changes of EGFR pathway markers in synovial sarcoma. J Surg Oncol 2011;103:773-781.

41 Baohua Y, Xiaoyan Z, Tiecheng Z, et al. Mutations of the PIK3CA gene in diffuse large B cell lymphoma. Diagn Mol Pathol 2008;17:159-165.

42 Kang S, Seo SS, Chang HJ, et al. Mutual exclusiveness between PIK3CA and KRAS mutations in endometrial carcinoma. Int J Gynecol Cancer 2008;18:1339-1343.

43 Kwon O, Jeong SJ, Kim SO, et al. Modulation of E-cadherin expression by K-Ras; involvement of DNA methyltransferase-3b. Carcinogenesis 2010;31:1194-1201.

44 Carragher LA, Snell KR, Giblett SM, et al. V600EBraf induces gastrointestinal crypt senescence and promotes tumour progression through enhanced CpG methylation of p16INK4a. EMBO Mol Med 2010;2: 458-471.

45 Hou P, Ji M, Xing M. Association of PTEN gene methylation with genetic alterations in the phosphatidylinositol 3-kinase/AKT signaling pathway in thyroid tumors. Cancer 2008;113:2440-2447. 